\title{
In vivo assessment of spinal cord elasticity using shear wave ultrasound in dogs
}

\author{
Amro Al-Habib, MD, FRCSC, MPH, ${ }^{1}$ Abdulrahman Albakr, MD, ${ }^{1}$ Abdullah Al Towim, MD, ${ }^{1}$ \\ Metab Alkubeyyer, MD, ${ }^{2}$ Abdullah Abu Jamea, PhD, ${ }^{2}$ Fahad Albadr, MD, ${ }^{2}$ \\ Abdelazeem A. Eldawlatly, MD, ${ }^{3}$ Tarek Kashour, MD, ${ }^{4}$ Hisham Alkhalidi, MD, ${ }^{5}$ and \\ Tariq Alzahrani, MD ${ }^{3}$ \\ ${ }^{1}$ Division of Neurosurgery, Department of Surgery, and Departments of ${ }^{2}$ Radiology, ${ }^{3}$ Anesthesia, ${ }^{4}$ Cardiac Sciences, and \\ ${ }^{5}$ Pathology, College of Medicine, King Saud University, Riyadh, Saudi Arabia
}

\begin{abstract}
OBJECTIVE Evaluation of living tissue elasticity has wide applications in disease characterization and prognosis prediction. Few previous ex vivo attempts have been made to characterize spinal cord elasticity (SCE). Recently, tissue elasticity assessment has been clinically feasible using ultrasound shear wave elastography (SWE). The current study aims to characterize SCE in healthy dogs, in vivo, utilizing SWE, and to address SCE changes during compression.
\end{abstract}

METHODS Ten Greyhound dogs (mean age 14 months; mean weight $14.3 \mathrm{~kg}$ ) were anesthetized and tracheally intubated, with hemodynamic and neurological monitoring. A 3-level, midcervical laminectomy was performed. SCE was assessed at baseline. Next, 8- and 13-mm balloon compressions were sequentially applied ventral to the spinal cord.

RESULTS The mean SCE was $18.5 \pm 7 \mathrm{kPa}$. Elasticity of the central canal, pia mater, and dura mater were $21.7 \pm 9.6$ $\mathrm{kPa}, 26.1 \pm 14.8 \mathrm{kPa}$, and $63.2 \pm 11.5 \mathrm{kPa}$, respectively. As expected, the spinal cord demonstrated less elasticity than the dura mater $(p<0.0001)$ and pia mater (trend toward significance $p=0.08)$. Notably, the $13-\mathrm{mm}$ balloon compression resulted in a stiffer spinal cord than at baseline $(233 \pm 73 \mathrm{kPa}$ versus $18.5 \pm 7 \mathrm{kPa}, \mathrm{p}<0.0001)$ and 8 -mm balloon compression (233 $\pm 73 \mathrm{kPa}$ versus $185 \pm 68 \mathrm{kPa}, \mathrm{p}<0.048)$.

CONCLUSIONS In vivo SCE evaluation using SWE is feasible and comparable to earlier reports, as demonstrated by physical sectioning of the spinal cord. The compressed spinal cord is stiffer than a free spinal cord, with a linear increase in SCE with increasing mechanical compression. Knowledge of the biomechanical properties of the spinal cord including SCE has potential implications for disease management and prognosis.

https://thejns.org/doi/abs/10.3171/2018.2.SPINE171195

KEYWORDS ultrasound; shear wave elastography; spinal cord compression

$\mathrm{M}$ ECHANICAL properties of bodily tissues are altered by different disease processes. ${ }^{15,34}$ Recognition of such changes through organ palpation during examination is integral to medical diagnosis. The discovery of solid lesions may be suggestive of malignancy. ${ }^{41}$ In addition to the current imaging methods that visualize lesions based on their anatomical characteristics or contrast enhancement, hard and soft tissues can be differentiated using radiology, through tissue elasticity assessment. ${ }^{33}$ Both ultrasound elastography (USE) and MR elastography (MRE) are currently used to assess the elasticity of superficial and deep tissues. ${ }^{31,41}$
USE has wide applications as a diagnostic tool in different organs. ${ }^{35}$ It is used to identify malignant breast lesions by defining the lesion's elasticity., ${ }^{4,13,38} \mathrm{~A}$ study has shown that USE for breast lesions is superior to B-mode ultrasound (US) and mammography, due to its greater sensitivity and specificity. ${ }^{19}$ Another study has suggested that USE, compared to conventional US, is more accurate and specific in assessing breast lesions. This could lead to a reduction in the number of unnecessary biopsies. ${ }^{26}$ USE is also used in liver disease assessment for lesion evaluation and the grading of fibrosis; thus, possibly limiting the need for more invasive tests. ${ }^{36}$ It is also used for monitoring the liver disease

ABBREVIATIONS MAP = mean arterial blood pressure; MEP = motor evoked potential; MRE = MR elastography; ROI = region of interest; SCE = spinal cord elasticity; SWE = shear wave elastography; USE = ultrasound elastography.

SUBMITTED October 30, 2017. ACCEPTED February 27, 2018.

INCLUDE WHEN CITING Published online July 20, 2018; DOI: 10.3171/2018.2.SPINE171195. 
progression and treatment response..$^{35}$ Moreover, elasticity assessment using USE and MRE has demonstrated promising diagnostic value for several other organs including thyroid, lymph nodes, heart, lung, muscles, spleen, kidneys, pancreas, uterus, and prostate. . $^{3,8,18,20-22,24,29,31,37}$

The assessment of CNS elasticity has been a subject of developing research. ${ }^{10,12,22,25,29-31,37,40,42,43}$ Characterization of brain elasticity has shown potential diagnostic value for many diseases including normal pressure hydrocephalus, Parkinson's disease, dementia, multiple sclerosis, and brain tumors, and the noninvasive characterization of intracranial pressure. . $16,17,29,32,37,42$ In one study, brain elasticity values measured using MRE were lower in Alzheimer's disease mice than in the control group, indicating that elasticity could be used as a biomarker for the diagnosis or grading the disease. ${ }^{28}$ Brain elasticity assessment using MRE could predict brain tumor stiffness and correlates with the qualitative assessment of surgeons. ${ }^{29}$ Integration of such assessments is important in planning surgical resections. ${ }^{22}$ Furthermore, intraoperative USE has also been used to identify and resect an epilepsy focus that was not apparent on preoperative MRI or intraoperative B-mode US. ${ }^{10}$

Current knowledge on spinal cord elasticity (SCE) is very limited. Elasticity of the spinal cord and pia mater was assessed previously in dogs by tissue sectioning and the application of tensile-loading method. ${ }^{40}$ The study demonstrated that the sources of elasticity of the dura mater were both elastic and collagenous fibers. ${ }^{39}$ Other studies have demonstrated that both pia mater and the dura mater are stiffer than the spinal cord. ${ }^{30,39,40}$ Overall, published studies on the subject are few and limited to physical experiments that were done ex vivo. They also lacked the demonstration of the effect of compression on SCE. Our current study aims at evaluating SCE in vivo. USE was utilized to assess the elasticity of the spinal cord, pia, and dura intraoperatively in anesthetized dogs after cervical laminectomy. The response of SCE during external compression was also assessed. The results of the current study may provide more insight into the biomechanical properties of the spinal cord and could have future implications for the diagnosis and prognosis of spinal cord diseases.

\section{Methods}

\section{Experiment Preparation and Procedure}

Ten Greyhound dogs weighing 8.6-20.0 kg (mean 14.3 $\mathrm{kg}$ ), comprising 6 males and 4 females, with a mean age of 14 months, were included in the study. The Institutional Ethics Review board approved the study protocol.

General anesthesia was induced with intramuscular ketamine and xylazine ( $2 \mathrm{mg} / \mathrm{kg}$ each). Tracheal intubation was then performed and followed by connecting the dogs to the respirator for volume-controlled ventilation with oxygen and sevoflurane-maintenance anesthesia. The dogs were fully anesthetized during the procedure. An intravenous line was inserted to deliver the medications, and an infusion of Ringer's lactate was started. Continuous intraoperative monitoring of the heart rate, blood pressure, mean arterial blood pressure (MAP), and oxygen saturation was performed. Intraoperative neurophysiological monitoring in the form of motor evoked potentials (MEPs) was used to assess the responses from both upper and lower limbs. Baseline MEPs were recorded prior to the beginning of the procedure.

The incision was done in the midline of the posterior neck area. After muscle dissection and posterior spine exposure, a 3-level midcervical laminectomy was performed, exposing the dura. US was then performed for anatomy and elasticity evaluation. At the end of the procedure, the animals were humanely euthanized, and mechanical ventilation was discontinued.

\section{Elasticity Evaluation and Quantification}

Elasticity of the spinal cord was studied using the SuperSonic Aixplorer ultrasound system (SuperSonic Imagine S.A.) coupled with a linear transducer array (4-15 MHz, SuperLinear 15-4). ${ }^{5}$ The SuperSonic US system measures tissue elasticity through the shear wave elastography (SWE) technique. ${ }^{45}$ The probe sends mechanical waves that propagate through the tissues. ${ }^{45}$ Shear waves are the transverse components of the transmitted waves. The speed of the waves is variable depending on tissue hardness. Therefore, measuring the speed of the shear waves provides an assessment of the tissue stiffness, presented in kilopascals. ${ }^{6}$ Since the probe, rather than the operator, is the source of the waves, SuperSonic SWE is less dependent on the operator, making the technique and results reproducible and quantitative..$^{14,45}$

Following laminectomy with the dura kept intact, the surgical field was filled with normal saline, avoiding air bubbles. The spinal cord and surrounding structures were assessed using the B-mode US (Fig. 1A). Subsequently, SCE assessment using SWE sequences was performed. During SWE image acquisition, it is necessary to avoid pressure by the US probe resulting in variability in elasticity results. SuperSonic US demonstrates if any pressure is applied by the probe, by measuring the elasticity in the extradural space where the irrigated fluid exists (Fig. 1).

Elasticity values were determined on the midline sagittal image for the spinal cord, central canal, pia mater, and dura mater. B-mode US imaging of the spinal cord was done in the sagittal midline plane showing the central canal (Fig. 1). The image was then immobilized by avoiding any movement for a few seconds, for SWE assessment. The image was then frozen and saved, and elasticity measurement was performed. Elasticity acquisition using SuperSonic SWE was performed, as previously described..$^{45}$ Tissue elasticity was superimposed on the B-mode US images as a color-scaled image; tissues exhibiting lower stiffness (lower values in $\mathrm{kPa}$ ) were displayed in blue, while tissues with higher stiffness were displayed in red (higher values in $\mathrm{kPa}$, Fig. 1). ${ }^{6} \mathrm{SCE}$ quantification was determined on 3 sagittal SWE image acquisitions for each dog. Within each acquisition, 2 to 3 random points representing the region of interest (ROI) were specified (Fig. 1A). Each ROI was defined by a sizable circle (the so-called Q-Box) that is built into the system; the operator would place the ROI where elasticity measurement was desired. The values of the mean, maximum, minimum, and standard deviation relevant to the elasticity of the $\mathrm{ROI}$ (in $\mathrm{kPa}$ ) would be dis- 


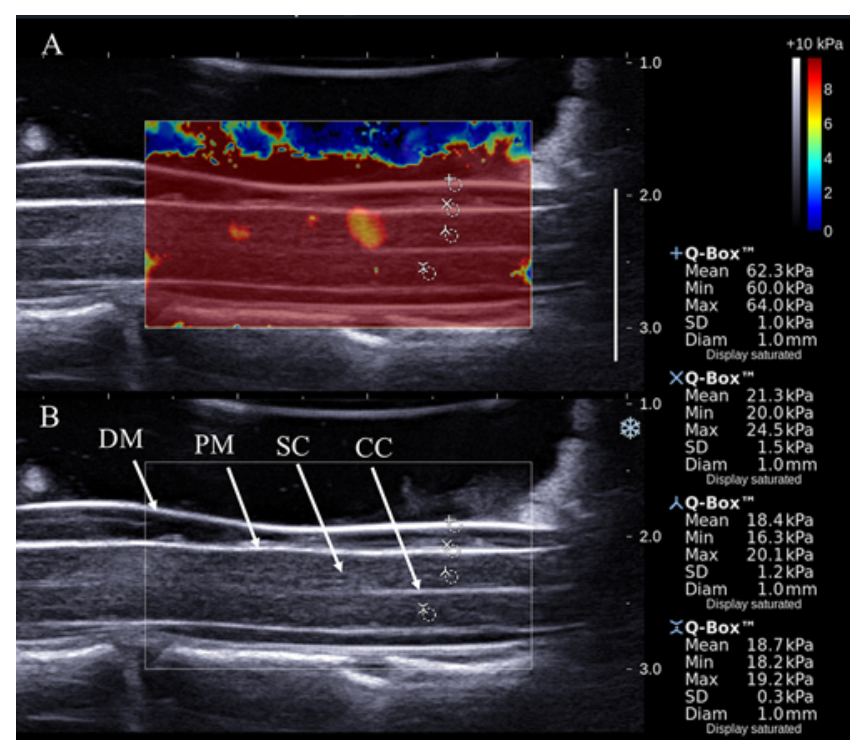

FIG. 1. Sagittal-view US image (A) of the spinal cord showing the elasticity map obtained using SWE, superimposed on a B-mode US image (B). Summary of SWE information for the selected ROI called Q-Box is displayed on the right side of the image. Each Q-Box is labeled with a specific symbol that addresses a specific ROI. In this case, (+ Q-Box) represents dura mater, $(\times \mathrm{Q}-\mathrm{Box})$ represents pia mater, and the other two $Q$-Boxes were selected randomly to represent the spinal cord. Data for each ROI are displayed on the screen, and they include the mean, maximum, minimum, and standard deviation of the measured elasticity. All the numbers are expressed in kilopascals $(\mathrm{kPa})$. For accurate evaluation, each selected ROI was determined using the smallest $Q$-Box circle $(1 \mathrm{~mm})$ in size. $\mathrm{CC}=$ central canal; $\mathrm{DM}=$ dura mater; $\mathrm{PM}=$ pia mater; $\mathrm{SC}=$ spinal cord. Figure is available in color online only.

played accordingly. For accurate evaluation of elasticity, we selected the smallest Q-Box circle $(1 \mathrm{~mm})$ to isolate the location of interest and avoid inclusion of adjacent tissues (Fig. 1A).

\section{Studying the Effect of Local Compression on the Elasticity of the Spinal Cord}

The second part of the study involved addressing the $\mathrm{SCE}$ changes in response to compression. A balloon catheter was introduced anterior to the spinal cord during the laminectomy exposure. The compression was carried out in two steps, starting with gradual inflation of the balloon to $8 \mathrm{~mm}$, followed by an increase to $13 \mathrm{~mm}$ of air with the assessment of SCE at each step of the compression (Fig. 2A-D). The balloon location was confirmed by B-mode US. SCE was assessed using the same SWE technique, at the maximum local site of compression, in addition to other points on the spinal cord distal to it (Fig. 3A and B).

During the entire procedure, neuromonitoring using MEPs in the upper and lower limbs indicated MEP levels at baseline prior to skin incision, after laminectomy, and before and after the compression.

\section{Statistical Analysis}

All statistical calculations, including Pearson correlation coefficients and paired-sample t-tests, were conducted using the Statistical Package for Social Science Software
(PC + version 19.0; SPSS, IBM). Descriptive statistics (mean $\pm \mathrm{SD}$, and $95 \% \mathrm{CI}$ ) were determined. A result was considered statistically significant if $\mathrm{p}<0.05$.

\section{Results}

\section{Elasticity of the Spinal Cord, Central Canal, Pia Mater, and Dura Mater}

Elasticity values of different tissues were demonstrated in Table 1. The spinal cord exhibited the least elasticity values, indicating that it is a softer structure compared to the dura mater, which is comparatively sturdier (Fig. 1, Table 1, p < 0.0001). Likewise, pia mater elasticity showed a higher value than that of the spinal cord; however, this difference demonstrated a trend toward significance $(\mathrm{p}=$ 0.08). Central canal elasticity did not exhibit a significant difference compared to the spinal cord $(p=0.228)$ or the pia mater $(p=0.162)$. There was no significant correlation between the subject's age, weight, heart rate, and SCE ( $\mathrm{p}=$ $0.307, \mathrm{p}=0.399, \mathrm{p}=0.906$, respectively).

During the procedure, the mean systolic blood pressure, diastolic blood pressure, and MAP were $115.7 \mathrm{~mm} \mathrm{Hg}, 76$ $\mathrm{mm} \mathrm{Hg}$, and $86.7 \mathrm{~mm} \mathrm{Hg}$ respectively. Interestingly, SCE was negatively correlated with the mean systolic blood pressure $(\mathrm{r}=-0.74, \mathrm{p}=0.013)$, diastolic blood pressure $(\mathrm{r}$ $=-0.72, \mathrm{p}=0.018)$, and MAP $(\mathrm{r}=-0.74, \mathrm{p}=0.014)$. This could be related to changes in the spinal cord fluid status with different hemodynamic values. It is well known that spinal cord blood flow changes with changing systemic blood pressure. ${ }^{23}$ Tissue elasticity evaluation is subject to change when the tissue vascularity changes, as previously shown in the case of liver. ${ }^{27}$ While recognizing that SCE changes with different hemodynamic values, it is beyond the scope of the current research to accurately define the fluid status of the spinal cord in response to changes in hemodynamic values and correlate that to SCE.

\section{Quantitative Assessment of SCE With Compression}

The local application of an 8-mm balloon compression on the spinal cord significantly increased the stiffness of the cord, as demonstrated by increased SCE values (mean $185 \pm 68 \mathrm{kPa}$ versus $18.5 \pm 7 \mathrm{kPa}, \mathrm{p}=0.004)$. Moreover, increasing the amount of balloon pressure to $13 \mathrm{~mm}$ led to an additional elevation in SCE values (mean $233 \pm 73$ $\mathrm{kPa}$ versus $18.5 \pm 7 \mathrm{kPa}, \mathrm{p}<0.0001)$. A 12-fold increase in the SCE value was demonstrated by the appearance of red color within the compressed area of the spinal cord on the SuperSonic SWE image (Fig. 3C). There was a significant difference between the results of SCE, considering the 2 levels of compression $(233 \pm 73 \mathrm{kPa}$ with $13-\mathrm{mm}$ balloon versus $185 \pm 68 \mathrm{kPa}$ with 8 -mm balloon, $\mathrm{p}<0.048$ ) (Fig. $3 \mathrm{C})$. The impact of the compression was not only limited to the site of compression. In fact, areas located distally, 1 to $2 \mathrm{~cm}$ in the cranial or caudal sides, were stiffer than the normal spinal cord $(18.5 \pm 7 \mathrm{kPa}$ versus $88.1 \pm 35.7 \mathrm{kPa}, \mathrm{p}$ $<0.0001$ ) (Fig. 3A and B).

Neuromonitoring was used to evaluate baseline neural function during spinal cord compression, and otherwise. Among the 10 subjects in the study, MEPs were not obtainable from the lower limbs since the beginning, in 2 subjects, due to technical difficulties. The assessment of 

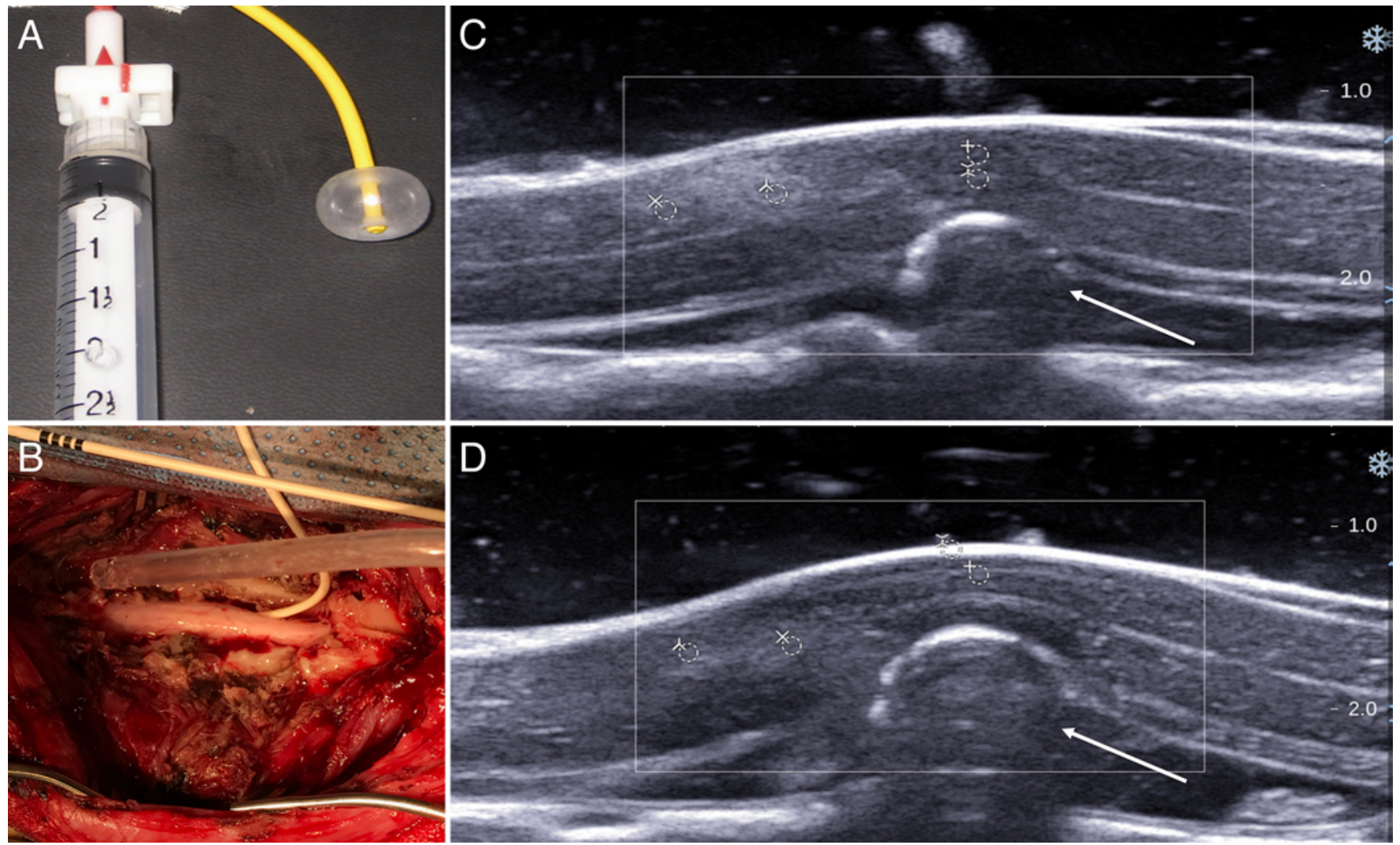

FIG. 2. A and B: The spinal cord is exposed to local compression by a balloon to measure changes in elasticity. C: B-mode US image demonstrating local compression of the spinal cord due to the balloon (arrow) located ventral to the cord. Inflation of the balloon was done in 2 steps with different amounts of pressure. The first step started with inflating the balloon to $8 \mathrm{~mm}$ of air with the assessment of SCE afterward. D: Subsequently, the amount of pressure in the balloon is increased to $13 \mathrm{~mm}$ of air followed by the assessment of spinal cord elasticity. Figure is available in color online only.

MEP changes during compression, in the remaining 8 subjects, revealed either maintained or slight MEP changes in 5 subjects $(62.5 \%)$ and complete loss of MEP signals in the remaining 3 subjects $(37.5 \%)$. With spinal cord compression, SCE values were higher in subjects who lost the MEP signals compared to those who maintained or demonstrated slight MEP changes (mean $264.4 \pm 61.5 \mathrm{kPa}$ versus $188.2 \pm 67.8 \mathrm{kPa}, \mathrm{p}=0.099$ ).

One of the dogs had a different focal area of higher echogenicity within the spinal cord, particularly in the area of the gray matter, as found using B-mode US (Fig. $4 \mathrm{~A}-\mathrm{C})$. Elasticity evaluation revealed that these abnormal focal areas were stiffer compared to the adjacent normallooking spinal cord, in the same dog (mean $64 \mathrm{kPa}$ versus $29 \mathrm{kPa} ; \mathrm{p}=0.04$ ). Histopathological examination of that segment, including the areas of increased echogenicity, showed mild edema of spinal cord tissue and foci of recent hemorrhage within the gray mater. No evidence of well-defined cyst or tumor was present. The findings could be related to inadvertent surgical trauma.

\section{Discussion}

\section{The Current Study}

The current study is the first to demonstrate SCE in vivo. In 1978, Tunturi published one of the earliest studies on measuring SCE in dogs, using the uniaxial tensile test. The elastic moduli of the spinal cord was estimated to be $16.8 \mathrm{kPa}$ and $11.9 \mathrm{kPa}$ at different loading modes. ${ }^{40}$ Two decades later, Ozawa et al. examined the mechanical properties of the spinal cord in rabbits using the tensile test and presented unique findings. ${ }^{30}$ In their report, the spinal cord with an intact pia had an elasticity value of $16 \mathrm{kPa}$, while it was $5 \mathrm{kPa}$ when the pia was removed. ${ }^{30}$ Therefore, they concluded that the pia mater tripled the elastic modulus, making the spinal cord stiffer. Furthermore, the pia mater was found to enhance the restoration of the cross-sectional shape of the spinal cord after the removal of compression. ${ }^{30}$ Our findings are in agreement with the findings of Tunturi and Ozawa et al., as a mean SCE value of $18.5 \mathrm{kPa}$ was found in our study, in addition to the result that the pia mater is stiffer than the spinal cord. Moreover, our elasticity measurements were performed while the animals were alive and the spinal cord was under normal physiological conditions, as evaluated by real-time neuromonitoring. This indicates that the current work is more relevant, and the results are more applicable due to the in vivo nature of experimentation. The current study was also the first to demonstrate an increased stiffness of the spinal cord with increasing 

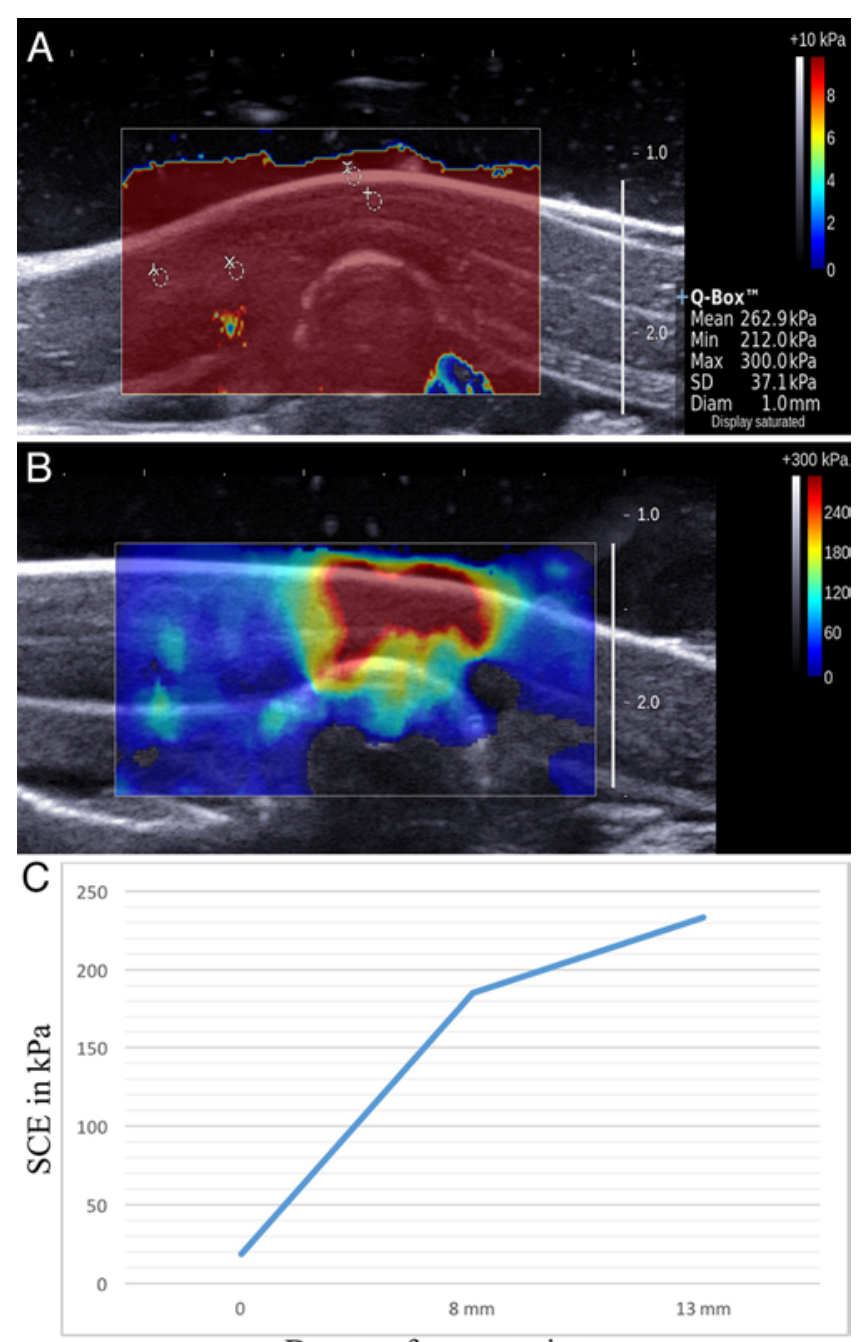

Degree of compression

FIG. 3. Changes in SCE in response to spinal cord compression. A: Summary of SWE values for the selected $\mathrm{ROI}$ at the compression site (+ Q-Box) is displayed on the right side of the image. The mean elasticity value for the compressed $\mathrm{ROI}$ is $262 \mathrm{kPa}$, as compared to the normal (free) spinal cord mean elasticity value of $18 \mathrm{kPa}$. B: Elasticity image of the compressed spinal cord with color-scale presentation. The compressed site demonstrates a red color, indicating increased stiffness (SCE 240 to $300 \mathrm{kPa}$ ) as presented in the scale at the right side of the image. Tissues located adjacent to the area of maximum compression exhibit an increased stiffness as demonstrated in blue (SCE $100 \mathrm{kPa}$ ). C: A linear relationship is demonstrated as increased SCE values with increasing degree of compression. Figure is available in color online only.

compression. Such a finding exhibits both diagnostic and prognostic significance in the assessment of different disease conditions affecting the spinal cord.

\section{Physics of Elasticity}

Elasticity is the inherent ability of the object to return to its original shape and size after deformation. ${ }^{41}$ The relationship between the applied force (stress or $\sigma$ ) and the resulting deformation (strain or $\varepsilon$ ) is presented as Young's modulus $\mathrm{E}(\mathrm{E}=\sigma / \varepsilon)$. The methods of tissue elasticity as-
TABLE 1. Elasticity values of different regions of the spinal cord using the SuperSonic SWE

\begin{tabular}{lcccc}
\hline \multirow{2}{*}{\multicolumn{1}{c}{ Location }} & \multicolumn{4}{c}{ Elasticity Values (kPa) } \\
\cline { 2 - 5 } & Mean & SD & Minimum & Maximum \\
\hline Spinal cord & 18.5 & 7 & 5.4 & 36.1 \\
\hline Central canal & 21.7 & 9.6 & 11.1 & 55.2 \\
\hline Pia mater & 26.1 & 14.8 & 14.1 & 69.3 \\
\hline Dura mater & 63.2 & 11.5 & 36.8 & 107 \\
\hline Compressed spinal cord* & 185 & 68 & 110 & 203 \\
\hline Compressed spinal cord† $^{*}$ & 233 & 73 & 178 & 300 \\
\hline
\end{tabular}

* Inflating the balloon with $8 \mathrm{~mm}$ of air.

† Inflating the balloon with $13 \mathrm{~mm}$ of air.

sessment using US depend on either strain or shear wave techniques. ${ }^{35,41}$ The strain method assesses elasticity by repeated manual compression from the transducer, measuring the amount of deformation relative to the normal surrounding tissue.." Using the "strain elastography" technique, Chakraborty et al. differentiated tumors from normal brain tissue by applying gentle pressure from the transducer on the brain to generate strain. ${ }^{9}$ A drawback of this technique, however, is the use of external force to generate strain. The produced force is not uniform and is subject to interoperator variability. ${ }^{11}$

In contrast, the shear wave technique does not produce deformation. SWE was first reported in 1998 as a new diagnostic method based on measuring the speed of travel of remotely induced shear acoustic waves through tissues. ${ }^{33,41}$ The shear waves are produced by a focused US beam and an ultrafast imaging approach at a very high frame rate (up to $5000 \mathrm{frames} / \mathrm{sec}$ ), which allows quantitative elasticity assessment of biological tissues in a single ultrasonic sequence. ${ }^{6,38}$ The measurement of the velocity of the shear wave propagation speed in soft tissues enables the creation of an elasticity map. ${ }^{6}$ A stiffness map is extracted through Young's modulus (E), or elasticity, in relation to shear wave speed (c) by the equation $\mathrm{E}=3 \mathrm{pc}^{2}$, where $\mathrm{p}$ is the tissue density $\left(\mathrm{kg} / \mathrm{m}^{3}\right){ }^{18,35}$ The speed of the shear waves is variable depending on the hardness of the tissues. ${ }^{4}$ Stiff tissues tend to have high speeds of shear waves producing higher elasticity values (in $\mathrm{kPa}$ ). For instance, it was demonstrated that malignant breast lesions, which exhibit higher tissue stiffness, show higher mean elasticity values on SWE US examination. ${ }^{45}$ Since SWE does not require tissue deformation, it is more suitable for elasticity assessment of the brain and the spinal cord, where tissue deformation is undesirable?

\section{Quantification of Tissue Elasticity}

Analogous to palpation in clinical assessment, SWE can differentiate tissues based on their stiffness. Moreover, since the velocity of the shear waves $(\mathrm{m} / \mathrm{sec})$ can be measured, quantification of tissue elasticity, or Young's modulus $(\mathrm{kPa})$, is possible. ${ }^{44}$ Quantification of elasticity is performed by selecting small circular areas (ROIs; $1 \mathrm{~mm}$ in the current research) in the target area and in several other locations for comparison. The displayed colors and 

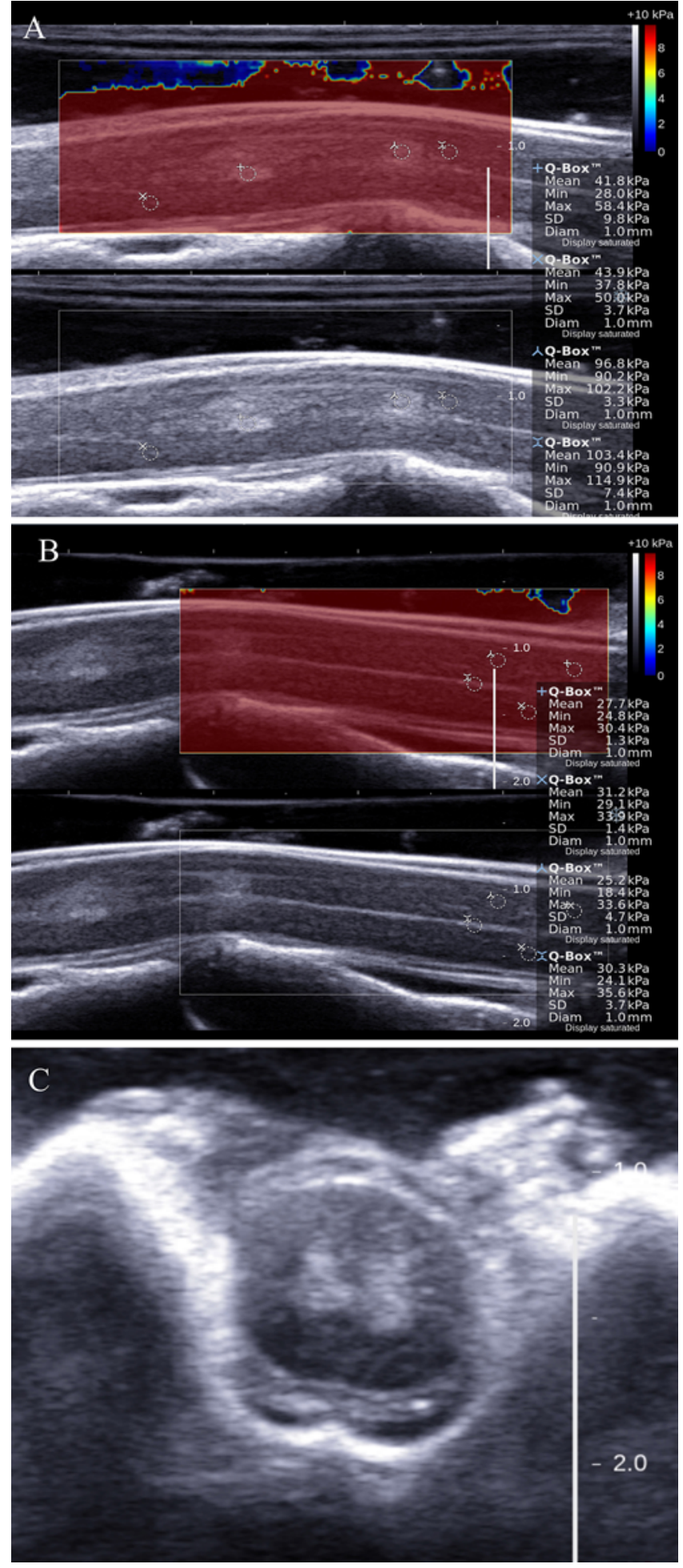

FIG. 4. B-mode US image (A), sagittal view, of a single dog demonstrating small rounded focal areas within the spinal cord exhibiting higher SWE values compared to image $(\mathbf{B})$ of the more rostral areas of the spinal cord (41.8-103.4 kPa versus 25.2-31.2 kPa). C: Axial US image of the spinal cord showing focal areas of increased echogenicity within the gray matter area. Figure is available in color online only. elasticity values indicate differences in elasticity of the lesion compared to that of other tissues. The elasticity value is provided in $\mathrm{kPa} .{ }^{44}$ Quantification of elasticity was useful in the current study where SCE demonstrated different values with variable degrees of balloon compression (Fig. 3).

\section{Elasticity Assessments of the Spinal Cord}

While US has been widely used in spine surgery for several decades, ${ }^{1}$ there is no previous report regarding its use to assess SCE. Furthermore, little is known about the mechanical properties of the spinal cord. Most available research has been conducted on ex vivo specimens. . $^{30,39,40}$ Such previous work has eliminated many factors that contribute to tissue stiffness in vivo, such as spinal cord vascularity and its fluid status. The current study addressed SCE in living and monitored subjects using USE for the first time. The agreement between the current results (SCE mean $18.5 \mathrm{kPa}$ ) and those of previous studies using physical sectioning of the cord is in favor of the utility of USE in such assessment. Together, these results are ideal for expanding the use of USE for SCE measurement in the future. $30,39,40$

The use of other imaging modalities for SCE measurement has been limited. Only a single report looked at the elasticity of the spinal cord using MRE in volunteers. ${ }^{25}$ Their findings demonstrated elasticity values of the superior portion of the spinal cord to be $12.1 \mathrm{kPa} .^{25}$

Previous research on the mechanical properties of the pia and dura mater resulted in significant variations in reported elasticity values. The variability was in part related to the methods of the study, leading to different conclusions on the actual elasticity values of the pia and dura mater. For instance, in one study, the elastic modulus of the pia mater was estimated to be $2300 \mathrm{kPa}$, whereas another study reported the elasticity value of the dura mater to start from $400 \mathrm{kPa}{ }^{30,39}$ In the current study, the dura mater was about twice as stiff as the pia mater. This finding corresponds well with the knowledge about the consistency of neuronal tissues.

Tissue compression changes the elastic properties of tissues. This was clearly demonstrated in the current study as a linear increase in the stiffness of the spinal cord with increasing spinal cord compression. In our study, the elasticity of the spinal cord increased in stiffness, 12 times, under the influence of compression. Our findings may underestimate the maximum effect of compression on SCE since the stiffness values in some cases reached the upper limit of the USE machine (300 kPa). On the other hand, it is necessary to correlate SCE values, with and without compression, to the neurological status of the subject. Some of the animals in the current study had preserved MEPs in the upper and lower limbs during compression. This corresponds to the clinical observation that some patients may have a preserved neurological status while undergoing spinal cord compression, as detected on MRI. However, it is necessary for future studies to evaluate whether quantifying SCE could predict future clinical deterioration. In the present work, we have shown the feasibility of USE for SCE evaluation. However, future work is needed for clinical correlation in order to validate the usefulness of this imaging technique and to demonstrate its clinical value. 
The current study should be interpreted within existing limitations. A larger sample size could provide more insight into understanding SCE. In the current study, we aspired to utilize many random areas of the spinal cord for precise elasticity evaluation. Within each subject, we obtained 3 different US-image acquisitions. Within each acquisition, we selected 2 to 3 random ROIs for elasticity evaluation, resulting in a minimum of 8 ROI points for measuring the SCE. An important limitation to note is regarding the generalizability of the results of animal studies to humans. Furthermore, the upper limit available in the SuperSonic SWE US machine is $300 \mathrm{kPa}$; some of the compressed SCE values reached the upper limit of 300 $\mathrm{kPa}$. We expect that these values could have been higher, although this could not be demonstrated in the US machine. While SCE measurement is possible using SWE US examination, USE requires the removal of bony and ligamentous structures. It is also possible that the remaining bone and ligament structures interfere with the sensitivity of the elasticity measurements. The need for bone removal such as in cases of compressive spinal pathology could affect the sensitivity of USE by freeing the spinal cord and, as a result, limiting the actual impact of compression on SCE values. More studies could be done to address SCE using MRE. Additionally, the current study did not address the white and gray matter of the spinal cord separately. Thus, it is necessary to conduct further experiments with a distinct evaluation of gray matter and white matter using USE. The current study also assessed the effect of acute compression on SCE. Future studies could develop methods to assess the effect of chronic compression on SCE.

\section{Conclusions}

The current study enhances the understanding of the biomechanical properties of the spinal cord. Using USE, normal SCE values were identified in dogs. Our results agree with basic anatomical knowledge that the dura mater has the highest elasticity, followed by the pia mater, and, lastly, the spinal cord. The central canal of the spinal cord did not exhibit a significant difference in elasticity compared to the spinal cord or pia mater. Moreover, the current study demonstrates significant changes in the spinal cord stiffness following the application of compression. The compressed spinal cord was much stiffer than the noncompressed cord. This should prompt future studies to utilize imaging techniques to provide reference values of SCE in humans. Currently, no modality other than USE is capable of defining tissue elasticity, in real time and intraoperatively. It is, however, limited by the surrounding bone and ligamentous structures.

The portable and economic features of this technique may exhibit potential future diagnostic and prognostic implications in various pathologies involving spinal cord compression. For example, evaluating SCE values in patients with cervical myelopathy or spinal cord injury may predict the functional outcome and the extent of recovery following surgery. Finally, USE may assist neurosurgeons during spinal surgery by accurately defining the region of interest. It may aid in detection, characterization, and definition of the boundaries of different spinal tumors, intraoperatively, thereby facilitating safer surgery. To our knowledge, this study is the first to confirm the feasibility of this technique for spinal cord evaluation. It, thus, supports future work to address technical issues and challenges associated with USE in the spinal cord, in addition to determining USE values that specify spinal cord biomechanical properties.

\section{Acknowledgments}

Dr. Albakr received a research grant from the Saudi Association of Neurological Surgery (SANS), Riyadh, Saudi Arabia. This study was also supported by the College of Medicine Research Center, Deanship of Scientific Research, King Saud University, Riyadh, Saudi Arabia.

\section{References}

1. Alaqeel A, Abou Al-Shaar H, Alaqeel A, Al-Habib A: The utility of ultrasound for surgical spinal decompression. Med Ultrason 17:211-218, 2015

2. Arani A, Min HK, Fattahi N, Wetjen NM, Trzasko JD, Manduca A, et al: Acute pressure changes in the brain are correlated with MR elastography stiffness measurements: initial feasibility in an in vivo large animal model. Magn Reson Med 79:1043-1051, 2018

3. Arda K, Ciledag N, Aktas E, Aribas BK, Köse K: Quantitative assessment of normal soft-tissue elasticity using shear-wave ultrasound elastography. AJR Am J Roentgenol 197:532-536, 2011

4. Athanasiou A, Tardivon A, Tanter M, Sigal-Zafrani B, Bercoff J, Deffieux T, et al: Breast lesions: quantitative elastography with supersonic shear imaging-preliminary results. Radiology 256:297-303, 2010

5. Bercoff J, Criton A, Cohen-Bacrie C, Souquet J, Tanter M, Gennison JL, et al: ShearWaveTM elastography. A new real time imaging mode for assessing quantitatively soft tissue viscoelasticity, in Proceedings of the 2008 IEEE Inernational Ultrasonics Symposium. Piscataway, NJ: IEEE, 2008, pp 321-324 (http://ieeexplore.ieee.org/stamp/stamp. jsp?arnumber $=4803626$ ) [Accessed April 5, 2018]

6. Bercoff J, Tanter M, Fink M: Supersonic shear imaging: a new technique for soft tissue elasticity mapping. IEEE Trans Ultrason Ferroelectr Freq Control 51:396-409, 2004

7. Bhatia K, Tong CS, Cho CC, Yuen EH, Lee J, Ahuja AT: Reliability of shear wave ultrasound elastography for neck lesions identified in routine clinical practice. Ultraschall Med 33:463-468, 2012

8. Bhatia KS, Tong CS, Cho CC, Yuen EH, Lee YY, Ahuja AT: Shear wave elastography of thyroid nodules in routine clinical practice: preliminary observations and utility for detecting malignancy. Eur Radiol 22:2397-2406, 2012

9. Chakraborty A, Bamber JC, Dorward NL: Preliminary investigation into the use of ultrasound elastography during brain tumour resection. Ultrasound 20:33-40, 2012

10. Chan HW, Pressler R, Uff C, Gunny R, St Piers K, Cross H, et al: A novel technique of detecting MRI-negative lesion in focal symptomatic epilepsy: intraoperative ShearWave elastography. Epilepsia 55:e30-e33, 2014

11. Chang JM, Won JK, Lee KB, Park IA, Yi A, Moon WK: Comparison of shear-wave and strain ultrasound elastography in the differentiation of benign and malignant breast lesions. AJR Am J Roentgenol 201:W347-56, 2013

12. Chauvet D, Imbault M, Capelle L, Demene C, Mossad M, Karachi C, et al: In vivo measurement of brain tumor elasticity using intraoperative shear wave elastography. Ultraschall Med 37:584-590, 2016 
13. Cho N, Moon WK, Park JS, Cha JH, Jang M, Seong MH: Nonpalpable breast masses: evaluation by US elastography. Korean J Radiol 9:111-118, 2008

14. Cosgrove DO, Berg WA, Doré CJ, Skyba DM, Henry JP, Gay $\mathrm{J}$, et al: Shear wave elastography for breast masses is highly reproducible. Eur Radiol 22:1023-1032, 2012

15. Duck FA: Physical Properties of Tissue: A Comprehensive Reference Book. London: Academic Press, 1990

16. ElSheikh M, Arani A, Perry A, Boeve BF, Meyer FB, Savica $\mathrm{R}$, et al: MR elastography demonstrates unique regional brain stiffness patterns in dementias. AJR Am J Roentgenol 209:403-408, 2017

17. Fattahi N, Arani A, Perry A, Meyer F, Manduca A, Glaser K, et al: MR elastography demonstrates increased brain stiffness in normal pressure hydrocephalus. AJNR Am J Neuroradiol 37:462-467, 2016

18. Ferraioli G, Tinelli C, Lissandrin R, Zicchetti M, Bernuzzi S, Salvaneschi L, et al: Ultrasound point shear wave elastography assessment of liver and spleen stiffness: effect of training on repeatability of measurements. Eur Radiol 24:1283-1289, 2014

19. Fischer T, Peisker U, Fiedor S, Slowinski T, Wedemeyer P, Diekmann F, et al: Significant differentiation of focal breast lesions: raw data-based calculation of strain ratio. Ultraschall Med 33:372-379, 2012

20. Gennisson JL, Muller M, Gabor P, Frydman R, Musset D, Tanter M, et al: Quantification of elasticity changes in the myometrium during labor using supersonic shear imaging: a feasibility study. Ultrasonics 56:183-188, 2015

21. Giovannini M, Thomas B, Erwan B, Christian P, Fabrice C, Benjamin E, et al: Endoscopic ultrasound elastography for evaluation of lymph nodes and pancreatic masses: a multicenter study. World J Gastroenterol 15:1587-1593, 2009

22. Hughes JD, Fattahi N, Van Gompel J, Arani A, Ehman R, Huston J III: Magnetic resonance elastography detects tumoral consistency in pituitary macroadenomas. Pituitary 19:286-292, 2016

23. Kise Y, Kuniyoshi Y, Inafuku H, Nagano T, Hirayasu T, Yamashiro S: Directly measuring spinal cord blood flow and spinal cord perfusion pressure via the collateral network: correlations with changes in systemic blood pressure. J Thorac Cardiovasc Surg 149:360-366, 2015

24. König K, Scheipers U, Pesavento A, Lorenz A, Ermert H, Senge T: Initial experiences with real-time elastography guided biopsies of the prostate. J Urol 174:115-117, 2005

25. Kruse SA, Kolipaka A, Manduca A, Ehman RL: Feasibility of evaluating the spinal cord with MR elastography, presented at the 17th Scientific Meeting, International Society for Magnetic Resonance in Medicine, 2009 (http://cds.ismrm. org/protected/09MProceedings/files/00629.pdf) [Accessed April 5, 2018]

26. Leong LC, Sim LS, Lee YS, Ng FC, Wan CM, Fook-Chong SM, et al: A prospective study to compare the diagnostic performance of breast elastography versus conventional breast ultrasound. Clin Radiol 65:887-894, 2010

27. Ling W, Lu Q, Lu C, Quan J, Ma L, Li J, et al: Effects of vascularity and differentiation of hepatocellular carcinoma on tumor and liver stiffness: in vivo and in vitro studies. Ultrasound Med Biol 40:739-746, 2014

28. Munder T, Pfeffer A, Schreyer S, Guo J, Braun J, Sack I, et al: MR elastography detection of early viscoelastic response of the murine hippocampus to amyloid $\beta$ accumulation and neuronal cell loss due to Alzheimer's disease. J Magn Reson Imaging 47:105-114, 2018

29. Murphy MC, Huston J III, Glaser KJ, Manduca A, Meyer FB, Lanzino G, et al: Preoperative assessment of meningioma stiffness using magnetic resonance elastography. J Neurosurg 118:643-648, 2013

30. Ozawa H, Matsumoto T, Ohashi T, Sato M, Kokubun S: Me- chanical properties and function of the spinal pia mater. $\mathbf{J}$ Neurosurg Spine 1:122-127, 2004

31. Pepin KM, Ehman RL, McGee KP: Magnetic resonance elastography (MRE) in cancer: technique, analysis, and applications. Prog Nucl Magn Reson Spectrosc 90-91:32-48, 2015

32. Perry A, Graffeo CS, Fattahi N, ElSheikh MM, Cray N, Arani A, et al: Clinical correlation of abnormal findings on magnetic resonance elastography in idiopathic normal pressure hydrocephalus. World Neurosurg 99:695-700, 700.e1, 2017

33. Sarvazyan AP, Rudenko OV, Swanson SD, Fowlkes JB, Emelianov SY: Shear wave elasticity imaging: a new ultrasonic technology of medical diagnostics. Ultrasound Med Biol 24:1419-1435, 1998

34. Sarvazyan AP, Skovoroda AR, Emelianov SY, Fowlkes JB, Pipe JG, Adler RS, et al: Biophysical bases of elasticity imaging, in Jones JP (ed): Acoustical Imaging. Boston: Springer, 1995, pp 223-240

35. Sigrist RMS, Liau J, Kaffas AE, Chammas MC, Willmann JK: Ultrasound elastography: review of techniques and clinical applications. Theranostics 7:1303-1329, 2017

36. Singh S, Venkatesh SK, Wang Z, Miller FH, Motosugi U, Low RN, et al: Diagnostic performance of magnetic resonance elastography in staging liver fibrosis: a systematic review and meta-analysis of individual participant data. Clin Gastroenterol Hepatol 13:440-451, 451.e1-451.e6, 2015

37. Streitberger KJ, Reiss-Zimmermann M, Freimann FB, Bayerl S, Guo J, Arlt F, et al: High-resolution mechanical imaging of glioblastoma by multifrequency magnetic resonance elastography. PLoS One 9:e110588, 2014

38. Tanter M, Bercoff J, Athanasiou A, Deffieux T, Gennisson JL, Montaldo G, et al: Quantitative assessment of breast lesion viscoelasticity: initial clinical results using supersonic shear imaging. Ultrasound Med Biol 34:1373-1386, 2008

39. Tunturi AR: Elasticity of the spinal cord dura in the dog. J Neurosurg 47:391-396, 1977

40. Tunturi AR: Elasticity of the spinal cord, pia, and denticulate ligament in the dog. J Neurosurg 48:975-979, 1978

41. Wells PN, Liang HD: Medical ultrasound: imaging of soft tissue strain and elasticity. J R Soc Interface 8:1521-1549, 2011

42. Wuerfel J, Paul F, Beierbach B, Hamhaber U, Klatt D, Papazoglou S, et al: MR-elastography reveals degradation of tissue integrity in multiple sclerosis. Neuroimage 49:2520 2525,2010

43. Xu ZS, Lee RJ, Chu SS, Yao A, Paun MK, Murphy SP, et al: Evidence of changes in brain tissue stiffness after ischemic stroke derived from ultrasound-based elastography. J Ultrasound Med 32:485-494, 2013

44. Youk JH, Gweon HM, Son EJ: Shear-wave elastography in breast ultrasonography: the state of the art. Ultrasonography 36:300-309, 2017

45. Youk JH, Son EJ, Park AY, Kim JA: Shear-wave elastography for breast masses: local shear wave speed $(\mathrm{m} / \mathrm{sec})$ versus Young modulus (kPa). Ultrasonography 33:34-39, 2014

\section{Disclosures}

The SuperSonic Imagine Aixplorer machine used in the study was provided by Gulf Medical Co. Ltd. (Saudi Arabia). However, there was no financial support provided by the company to any of the authors. Moreover, the supporting company did not have input into the study design, methodology, results interpretation, or conclusions.

The authors report no conflict of interest concerning the materials or methods used in this study or the findings specified in this paper. 


\section{Supplemental Information}

Previous Presentations

A portion of this work was presented in oral format at the The 16th Annual Surgery Research Day, King Saud University, Riyadh, Saudi Arabia, April 2017.

\section{Author Contributions}

Conception and design: Al-Habib, Albakr, Abu Jamea, Albadr, Eldawlatly, Kashour. Acquisition of data: all authors. Analysis and interpretation of data: all authors. Drafting the article: AlHabib, Albakr. Critically revising the article: Al-Habib, Albakr,
Alkubeyyer, Eldawlatly, Kashour, Alkhalidi. Reviewed submitted version of manuscript: all authors. Approved the final version of the manuscript on behalf of all authors: Al-Habib. Statistical analysis: Albakr. Administrative/technical/material support: Al-Habib, Albakr, Al Towim, Alkubeyyer, Abu Jamea, Eldawlatly, Kashour. Study supervision: Al-Habib.

\section{Correspondence}

Amro F. Al-Habib: College of Medicine, King Saud University, Riyadh, Saudi Arabia. amro.alhabib@gmail.com. 